\author{
Alterstice \\ Revue internationale de la recherche interculturelle \\ International Journal of Intercultural Research \\ Revista International de la Investigacion Intercultural
}

\title{
Dépister et orienter les personnes d'origine étrangère usant de violence au sein du couple : un défi pour les professionnels du champ social
}

\section{Susanne Lorenz et Christophe Fluehmann}

Volume 3, numéro 2, 2013

Violence conjugale et diversité culturelle

URI : https://id.erudit.org/iderudit/1077522ar

DOI : https://doi.org/10.7202/1077522ar

Aller au sommaire du numéro

Éditeur(s)

Alterstice

ISSN

1923-919X (numérique)

Découvrir la revue

Citer cet article

Lorenz, S. \& Fluehmann, C. (2013). Dépister et orienter les personnes d'origine étrangère usant de violence au sein du couple : un défi pour les professionnels du champ social. Alterstice, 3(2), 67-80. https://doi.org/10.7202/1077522ar

\section{Résumé de l'article}

En Suisse comme ailleurs, la violence au sein du couple s'inscrit comme un grave problème de société. Notre pays a accru le soutien aux victimes et aux programmes spécialisés pour auteurs de violence. Il poursuit l'objectif d'assurer durablement la sécurité des victimes dans le cadre d'un dispositif formalisé, intégrant une approche pluridisciplinaire. Cet article présente le point de vue de professionnels travaillant au sein d'institutions sociales susceptibles d'orienter les personnes usant de violence vers les groupes thérapeutiques et expose les résultats collectés lors de groupes de discussion. Les participants sont d'avis que l'intervention auprès des personnes usant de violence reste difficile dans un contexte non spécialisé, et l'est encore davantage si ces personnes sont d'origine étrangère. Nous abordons également leurs appréhensions lors du dépistage et de l'orientation des personnes d'origine étrangère usant de violence conjugale, et notamment leurs difficultés à prendre position : ils redoutent souvent que leur discours soit jugé discriminatoire lorsqu'ils mettent en rapport l'origine socioculturelle et la violence au sein du couple. Les participants aux groupes de discussion soulignent la nécessité de renforcer les collaborations avec les milieux associatifs, de recourir à des mesures adaptées aux membres des communautés étrangères et de fournir un accès aux groupes thérapeutiques. Notre article met en évidence l'impérieuse nécessité de développer des outils de détection intégrant la composante liée à l’origine socioculturelle. 


\title{
ज alterstice \\ ARTICLE THÉMATIQUE \\ Dépister et orienter les personnes d'origine étrangère usant de violence au sein du couple : un défi pour les professionnels du champ social
}

\author{
Susanne Lorenz ${ }^{1}$ et Christophe Fluehmann ${ }^{2}$
}

\section{Résumé}

En Suisse comme ailleurs, la violence au sein du couple s'inscrit comme un grave problème de société. Notre pays a accru le soutien aux victimes et aux programmes spécialisés pour auteurs de violence. Il poursuit l'objectif d'assurer durablement la sécurité des victimes dans le cadre d'un dispositif formalisé, intégrant une approche pluridisciplinaire. Cet article présente le point de vue de professionnels travaillant au sein d'institutions sociales susceptibles d'orienter les personnes usant de violence vers les groupes thérapeutiques et expose les résultats collectés lors de groupes de discussion. Les participants sont d'avis que l'intervention auprès des personnes usant de violence reste difficile dans un contexte non spécialisé, et l'est encore davantage si ces personnes sont d'origine étrangère. Nous abordons également leurs appréhensions lors du dépistage et de l'orientation des personnes d'origine étrangère usant de violence conjugale, et notamment leurs difficultés à prendre position : ils redoutent souvent que leur discours soit jugé discriminatoire lorsqu'ils mettent en rapport l'origine socioculturelle et la violence au sein du couple. Les participants aux groupes de discussion soulignent la nécessité de renforcer les collaborations avec les milieux associatifs, de recourir à des mesures adaptées aux membres des communautés étrangères et de fournir un accès aux groupes thérapeutiques. Notre article met en évidence l'impérieuse nécessité de développer des outils de détection intégrant la composante liée à l'origine socioculturelle.

\section{Rattachement des auteurs \\ ${ }^{1}$ Haute école spécialisée Suisse occidentale (HES-SO) Valais, Sion, Suisse; ${ }^{2}$ Haute école fribourgeoise de travail social (HEF-TS), Givisiez, Suisse.}

\section{Correspondance}

susanne.lorenz@hevs.ch

\section{Mots clés}

violence au sein du couple; migration; personnes auteures de violence; prévention; dépistage; intervenants sociaux

\section{Pour citer cet article :}

Lorenz, S. et Fluehmann, C. (2013). Dépister et orienter les personnes d'origine étrangère usant de violence au sein du couple : un défi pour les professionnels du champ social. Alterstice, 3(2), 67-80. 


\section{Introduction}

Notre société moderne se caractérise par des flux migratoires importants. Si les personnes en contexte migratoire ne constituent pas un ensemble homogène, force est de constater que certains groupes sont surreprésentés parmi les bénéficiaires de prestations sociales. En 2010, 5,2 \% de la population d'origine étrangère résidant en Suisse bénéficiaient de ces prestations, contre $2,2 \%$ de la population suisse (OFAS, 2012). Concernant le suivi par un service social, une analyse de dossiers fait état, dans 1 situation sur 7, de violences commises par l'un des partenaires (Seith, 2003). Romito (2003) estime que la proportion de victimes s'adressant à un service social se situe à $11 \%$. Ce premier constat nous conduit à penser que les intervenants dans le champ social sont plus que probablement confrontés, dans leur pratique professionnelle, à des personnes concernées par la violence au sein du couple et ayant un parcours migratoire.

En Suisse, le lien entre le parcours migratoire et l'adoption de comportements violents soulève des discussions récurrentes, notamment en raison d'une surreprésentation des populations étrangères dans certaines statistiques. Cette thématique, bien qu'assez récente, fait l'objet de nombreux débats, notamment pour améliorer le statut des personnes victimes migrantes et les politiques migratoires.

Notre étude avait pour but de mettre en évidence les mesures de prévention primaire, secondaire et tertiaire à l'égard des auteurs d'actes de violence au sein du couple. Nous exposons plus spécifiquement dans cet article le point de vue des professionnels du champ social susceptibles d'identifier des situations de violence et d'orienter les auteurs de ces actes vers une intervention spécialisée. Nous présenterons les résultats concernant les représentations de ces professionnels au sujet des mesures de prévention, de détection et d'orientation, qui font partie intégrante du dispositif d'intervention auprès des personnes à l'origine de violence au sein du couple. Nous concluons sur l'importance des outils de détection pour améliorer l'accessibilité de ces personnes aux programmes d'intervention spécialisée lorsqu'elles sont d'origine étrangère.

\section{De la violence...}

La violence au sein du couple correspond à un « ensemble de comportements, de paroles ou de gestes agressifs, brusques et répétés » (Broué et Guévremont, 1999, p. 18) à l'égard du ou de la partenaire (ou de ses biens). Dans le cadre de ce type de relation "basé[e] sur le contrôle et la domination " (Hirigoyen, 2009, p. 24), l'un des partenaires exerce son pouvoir sur l'autre au travers d'actes visant à atteindre son intégrité et à lui infliger des souffrances physiques, sexuelles ou psychiques (Gillioz, De Puy et Ducret, 1997; Hirigoyen, 2005; Johnson, 2005; Silvestre, Heim et Christen, 1999). Ces actes permettent à la personne auteure de se repositionner dans des situations quotidiennes qu'elle vit comme menaçantes et disqualifiantes (Black, Schumacher, Smith et Heyman, 1999; Lorenz et Bigler, 2013; Mayer, 2007). Ce processus fonctionne selon un cycle dynamique et répétitif (Walker, 2000), qui tend à se répéter de manière de plus en plus violente (Collectif ViFa, 2000). Cette violence se distingue en cela du conflit, qui consiste en une opposition d'intérêts dans le but de faire valoir son opinion sur un sujet donné, " [...] sans que la peur détermine qui sera le gagnant » (Paradis, 2012, p. 20).

\section{... et de la prévalence du phénomène}

La violence au sein du couple est un problème majeur de santé publique (Krug, Dahlberg, Mercy, Zwi et LozanoAscencio, 2002). Ces actes sont une cause importante d'invalidité et de mortalité (Garcia Moreno, 2003). Ce phénomène est loin d'être marginal en Suisse. Il engendre des coûts de près de 400 millions de francs suisses par an (Godenzi et Yodannis, 1999). Les victimes en sont le plus souvent des femmes (Rossel, Sorenti et Jaquier, 2007). Ainsi, 1 femme sur 5 est victime de violences physiques ou sexuelles durant sa vie de couple, dont $6 \%$ au cours des derniers 12 mois précédant l'enquête (Gillioz et collab., 1997).

Les données actuelles qui mettent en perspective le recours à la violence au sein du couple et l'appartenance nationale renvoient à des constats contradictoires. Les études de prévalence réalisées auprès de femmes vivant en Suisse ne permettent pas d'observer un risque plus élevé de subir des violences lorsque l'un des partenaires est de 
nationalité étrangère (Gillioz et collab., 1997; Gloor et Meier, 2004), et ce d'autant moins lorsque les facteurs socioéconomiques sont pris en compte (Killias, Simonin et De Puy, 2005).

À l'inverse, diverses statistiques font preuve d'un lien possible entre appartenance nationale et violence au sein du couple. Ainsi, les victimes et les auteurs de violence conjugale issus de communautés étrangères sont surreprésentés au niveau des interventions policières (Jaquier, 2008; Steiner, 2004). La probabilité d'être victime d'un homicide ou d'une tentative d'homicide par le partenaire est 2,5 fois plus élevée pour les femmes d'origine étrangère (Zoder, 2008). Les données provenant des centres d'accueil pour femmes victimes (Belser, 2005) et d'une unité médicale spécialisée dans les soins en situation de violence (Burquier, Hoffner, Romain et Mangin, 2009) tendent à confirmer la surreprésentation des victimes issues de communautés étrangères.

Ces constats s'expliquent de différentes manières. Appréhender du point de vue statistique le lien possible entre nationalité et violence dans les relations intimes se heurte à la difficulté d'accéder à des données probantes (Crenshaw, 2005; Jaquier, 2008) et à l'opérationnalisation de la variable " étranger ". Cette dernière s'exprime fréquemment par une opposition entre les personnes avec un passeport suisse et celles qui en sont dépourvues, et elle ne tient donc pas compte des multiples dimensions qui caractérisent le statut de migrant (autorisation de séjour, niveau de formation, degré d'intégration, etc.). II faut également mettre en perspective ces observations chiffrées avec les modalités d'enregistrement des cas, les pratiques des professionnels dans ce champ ainsi que les stratégies de (non)dénonciation de la part des victimes (Killias, 2002). Plusieurs hypothèses émises en référence au statut socioéconomique des personnes migrantes peuvent expliquer la surreprésentation des groupes de migrants dans les statistiques officielles (moyens financiers limités, faible accès au réseau de soins, réseau social restreint, etc.; Belser, 2005; Burquier et collab., 2009; Crenshaw, 2005).

Lors des enquêtes de victimisation, certains groupes sociaux particulièrement défavorisés sont difficilement atteignables, dont les migrants. La représentativité de ces groupes n'est ainsi pas assurée. Belser (2005) pose l'hypothèse que le nombre d'actes de violence subis et commis par des personnes d'origine étrangères se situe entre le résultat des études de prévalence et celui des statistiques policières.

\section{Le parcours migratoire, facteur de vulnérabilité}

Le processus migratoire s'inscrit dans une double violence : celle précédant le départ (Rojas-Viger, 2008) et celle liée à d'importants changements consécutifs à l'arrivée dans la nouvelle terre d'accueil. Dans la période qui suit leur arrivée, les personnes migrantes doivent s'adapter à un nouvel environnement, marqué par un changement de culture, la perte du réseau social, des difficultés financières, etc. (Bureau fédéral de l'égalité entre hommes et femmes, 2012).

Une trajectoire de vie marquée par la migration peut mettre à mal la cellule familiale, alors même que la matrice familiale devrait rester intacte pour offrir l'énergie nécessaire à la construction d'une nouvelle identité dans le pays d'accueil (Batista Wiese, Van Dijk et Seddik, 2009). À la suite de la rupture avec le modèle familial traditionnel et du repositionnement des membres consécutifs à des changements de statuts et à une redéfinition des rapports de pouvoir, cette matrice évolue (Rojas-Viger, 2008). S'en suit un décalage avec le système de croyances et de comportements transmis intergénérationnellement, qui permet d'appréhender et d'organiser les relations interpersonnelles (Lewis et Ippen, 2004, cités par Batista Wiese et collab., 2009). Ce processus peut créer des tensions face auxquelles la violence relationnelle devient une stratégie parmi d'autres pour maintenir l'état originel de la matrice.

Le parcours migratoire représente en cela un facteur de vulnérabilité. Ce n'est pas le rapport d'inégalité entre partenaires qui est en jeu, mais bien le fait que la redistribution des rapports de pouvoir n'est plus acceptée par l'un des deux (Gillioz et collab., 1997). On peut donc supposer que la cause de la violence dans le couple se situe avant tout dans le cumul des difficultés associées au parcours migratoire (Egger et Schär Moser, 2008; Jaspard, 2005). 


\section{Les mesures à l'égard des personnes usant de violence au sein du couple}

En Suisse, diverses dispositions légales visent à dissuader le recours à la violence, à assurer durablement la sécurité des victimes ou encore à responsabiliser les auteurs d'actes de violence (Mösch Payot, 2008; Schwander, 2006). Les adaptations réalisées depuis 2004, la poursuite d'office et l'éloignement du domicile contribuent à augmenter les contraintes envers les personnes usant de violence au sein du couple. Ce lien entre la répression et la diminution durable de la violence à l'égard du partenaire ne fait toutefois pas l'unanimité. L'efficacité des réponses punitives dépend principalement de l'insertion professionnelle et de l'engagement dans le couple, et on constate que les incarcérations ou les éloignements ne garantissent pas durablement la sécurité des victimes (Myers, 1996) : la cessation des violences ne dure que le temps durant lequel la sanction est redoutée (Saunders et Parker, 1989). Les arrestations peuvent également provoquer des effets pervers, comme le sentiment de la part du partenaire violent d'être injustement traité (Séverac, 2009).

Il nous paraît donc essentiel que la personne à l'origine d'actes de violence conjugale s'engage dans une démarche thérapeutique. C'est dans cette perspective, et pour améliorer le dispositif de lutte contre la violence au sein du couple, que nous considérons les interventions spécialisées auprès d'elle comme complémentaires aux mesures de soutien et de protection de la victime ainsi qu'à l'arsenal législatif répressif. Loin de se substituer à de telles réponses sociales, ces services proposent un travail de responsabilisation et d'intégration de l'interdit du passage à l'acte. Ces réponses diversifiées permettent d'appréhender les attitudes violentes dans leur globalité et leur complexité, en tant que fruits de l'interaction entre plusieurs systèmes individuels et environnementaux étroitement imbriqués (Krug et collab., 2002).

\section{Profil des participants à des programmes spécialisés pour auteurs d'actes de violence conjugale}

Egger (2008) a répertorié pour la Suisse 27 organismes qui offrent des prestations spécialisées dans ce domaine, qui s'adressent plutôt aux hommes. Ces services proposent principalement des séances de groupes dirigées par des équipes mixtes. Ils sont localisés en zones urbaines. Les personnes qui contactent un service de ce type viennent à priori de leur plein gré, la contrainte exercée par une instance sociojudiciaire s'avérant plutôt l'exception (Egger, 2008). Définir leur profil s'avère difficile, car elles proviennent de toutes les couches socioculturelles (Gondolf, 2003). Elles se distinguent toutefois par la maîtrise de la langue locale et par leur engagement durable envers leur couple et envers leur rôle de parent (Lorenz et Anglada, 2011; Rondeau, Brodeur et Carrier, 2001; Séverac, 2009). En effet, l'insertion professionnelle incite non seulement à entamer un traitement, mais aussi à l'achever (Mathieu, Bélanger et Bribois, 2006; Rondeau et collab., 2001). À l'inverse, les personnes sans emploi régulier et prestataires des assurances sociales cessent le plus souvent leur participation au groupe (Barz et Helffrich, 2006).

Ces constats posent la question de la représentativité des personnes demandeuses d'aide par rapport à l'ensemble celles ayant recours à des comportements violents, et ce d'autant plus que plusieurs facteurs déterminent l'accessibilité à ces programmes (Rondeau et collab., 2001).

\section{ViFa, une intervention spécialisée dans le Canton de Vaud}

Le service ViFa, de la Fondation Jeunesse et Familles, intervient auprès de personnes usant de violence au sein du couple. Le modèle d'intervention, inspiré du programme d'Option, prévoit des entretiens individuels puis des rencontres de groupes animées par des équipes professionnelles spécialement formées. L'évaluation de ce programme a mis en évidence que les hommes qui achèvent ce programme développent des compétences en matière de responsabilisation et de dévoilement (Lorenz et Bigler, 2013).

Dans ce programme, $80 \%$ des participants masculins sont originaires de Suisse. Ceux d'origine étrangère doivent être considérés comme intégrés : ils vivent en Suisse depuis de nombreuses années et témoignent d'un minimum de maîtrise du français (Lorenz et Anglada, 2011). Les migrants sont ainsi sous-représentés (Anglada et Lorenz, 2007), pour plusieurs raisons. Nous pensons, notamment, à un accès rendu difficile par une maîtrise insuffisante de la langue, au manque d'information quant aux prestations ou à la crainte d'être expulsé ou stigmatisé. Damba et Anglada (2005) sont d'avis que l'engagement dans le groupe thérapeutique est difficile pour un migrant en raison de la représentation qu'il se fait de lui-même et de l'absence de contrainte que son ou sa partenaire est susceptible

Alterstice - Revue Internationale de la Recherche Interculturelle, vol. 3, $n^{\circ} 2$ 
d'exercer. Ce dernier aspect semble jouer un rôle indéniable : les victimes migrantes « ne peuvent pas utiliser la menace d'une séparation ou d'un divorce pour les amener dans une démarche de changement " (Damba et Anglada, 2005, p. 56) car leur autorisation de séjour dépend fréquemment de leur partenaire. Dans ce contexte, le statut administratif de certains migrants freine la démarche de changement.

\section{Identifier les pratiques professionnelles à l'égard des auteurs d'actes de violence conjugale}

Le canton de Vaud s'est doté d'un dispositif de prévention et de lutte contre les violences au sein du couple, dont la Commission cantonale de lutte contre la violence domestique (CCLVD), présidée par le Bureau de l'égalité entre les femmes et les hommes. Cette Commission s'est donnée pour mission de coordonner l'action des principales instances administratives et judiciaires de l'État et des organisations privées actives dans la lutte contre la violence domestique. Elle propose diverses mesures de prévention et d'intervention dans ce domaine. Selon Hofner, Stalder, Pedevilla, Detraz et Saturno (2011), plusieurs mesures ciblées ont vu le jour au cours des dix dernières années, mais les auteures soulignent la nécessité de renforcer les prestations pour les personnes usant de violence au sein du couple. La CCLVD a mandaté une étude sur les pratiques dans ce domaine, souhaitant un état des lieux des mesures actuelles de prévention et d'intervention.

\section{L'échantillon}

L'étude à l'origine des résultats présentés ici a été mandatée par la CCLVD. Cette étude exploratoire, réalisée entre novembre 2010 et avril 2011, a passé en revue les mesures de prévention et d'intervention ciblant les personnes usant de violence au sein du couple. Elle s'est intéressée aux mesures de prévention primaire, secondaire et tertiaire qui, aux yeux des professionnels du champ social recontrés, ont donné satisfaction et à celles qui restent à améliorer ou à développer.

Lors de cette étude, nous avons recueilli l'opinion de 41 personnes travaillant dans différents champs : judiciaire, psychosocial, médical, etc. Parmi elles, 30 professionnels, relevant de 26 services ou institutions, proposaient un accompagnement psychosocial ${ }^{1}$. Leur mandat prioritaire ne s'attachait pas donc nécessairement à l'intervention auprès de personnes usant de violence au sein du couple : la majorité des services pouvait plutôt être considérée comme de nature polyvalente, dans la mesure où les bénéficiaires de prestations présentent des problématiques multiples et que l'action socioprofessionnelle comprend plusieurs formes de soutien (aide sociale, soutien à l'insertion socioprofessionnelle, traitement des dépendances, etc.). Les intervenants rencontrés étaient donc susceptibles d'être confrontés à des situations de violence relationnelle, d'avoir à offrir des mesures de prévention primaire en institution ou encore d'orienter vers des services spécialisés.

\section{La méthode}

Nous avons retenu la méthode du groupe de discussion, qui offre aux participants la possibilité de donner leur avis sur un sujet précis et en même temps de réagir aux propos émis par les autres. Cette technique permet de recueillir des points de vue diversifiés, des idées innovantes et des renseignements utiles pour saisir les besoins et les attentes des professionnels. Les contenus émis sont considérés comme des énoncés spontanés valides (Flick, 2000). Afin de centrer les échanges sur la perception du dispositif de prévention et d'intervention auprès des personnes usant de violence au sein du couple, le déroulement des séances de groupe a été structuré selon la méthode SEPO (Succès-Échecs-Potentialités-Obstacles, voir Acheroy et Hadjaj-Castro, 2006). Cet outil permet d'organiser les expériences relatées à l'aide d'une matrice qui comporte deux axes, l'un temporel (rétroactif et prospectif) et l'autre qualitatif (les interventions considérées en tant que "bonnes pratiques » et celles décrites comme points faibles du système). Les propos émis lors des groupes de discussion ont été synthétisés dans un tableau. Concrètement, nous avons procédé en trois temps: identification des succès et des échecs sur l'axe rétroactif, formulation des potentialités à développer et des obstacles à prendre en compte sur l'axe prospectif et enfin désignation des mesures à développer prioritairement.

\footnotetext{
${ }^{1}$ La répartition de leur champ d'action est la suivante : interventions auprès de couples et de familles ( $\left.n=11\right)$, soutien en cas de violence $(n=6)$, accès à l'aide sociale $(n=4)$, développement de mesures d'insertion professionnelle $(n=4)$ ou de politique publique, notamment dans les champs de la violence familiale $(n=4)$ et du soutien à la population migrante $(n=1)$.
} 


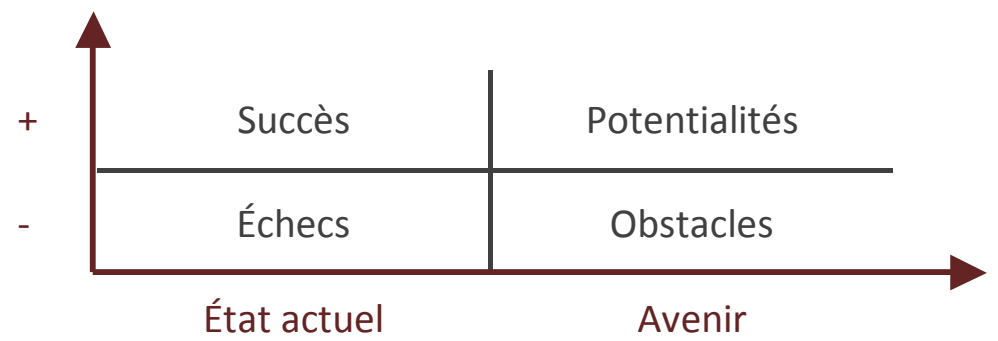

Figure 1. Tableau SEPO présenté aux participants des groupes de discussion

Le contenu a été analysé sur la base des propos tenus lors des rencontres ainsi que sur celle des idées figurant dans la matrice du SEPO puis il a été intégré au tableau de synthèse. Notre objectif a été de mettre en évidence le sens attribué par les participants aux différentes interventions et collaborations. Ce fonctionnement a permis de distinguer les discours des différents groupes professionnels et de rassembler ceux des 30 intervenants sociaux touchant les pratiques caractéristiques de leur champ professionnel : la prévention, la détection et l'orientation. Les résultats présentés ici se réfèrent aux discours de ces intervenants.

\section{Des difficultés à plusieurs niveaux}

De manière générale, les intervenants sociaux œuvrant au sein de services non spécialisés se disent de plus en plus conscients de l'existence de situations de violences relationnelles grâce aux pratiques de dépistage, qui se sont indéniablement améliorées pour les victimes. La plupart estiment que si le programme vaudois pour personnes usant de violence au sein du couple complète les consultations de couple ou de famille et les entretiens individuels, l'intervention spécialisée auprès de ces personnes demeure encore l'exception. Concernant le travail avec les personnes d'origine étrangère, les participants mentionnent un certain malaise ainsi que diverses restrictions dans leur intervention.

\section{Un manque de ressources}

Le manque de ressources et d'information joue un rôle déterminant : certains décrivent "le degré d'information sur les prestations destinées aux auteurs, respectivement migrants [comme] faible dans les institutions ». Ainsi, il semble qu'un seul acteur du réseau professionnel soit engagé spécifiquement dans le travail avec les victimes d'origine étrangère. Le réseau professionnel, non spécialisé dans l'intervention au niveau des violences familiales, dit toutefois ne pas identifier clairement le mandat de cette association. L'absence d'opportunités de coopération résulte apparemment de la méconnaissance des prestations pour personnes usant de violence au sein du couple offertes par cette association, ainsi que du manque de ressources. La collaboration avec d'autres milieux associatifs œuvrant auprès de communautés étrangères s'avère également ardue, quelques participants citant une certaine méfiance au moment d'élaborer ou de réaliser des projets de prévention orientés vers les personnes usant de violence au sein du couple et relevant de ces communautés. La crainte de la stigmatisation de ces personnes freine, notamment, la diffusion d'information auprès des diverses communautés.

Au-delà de la collaboration, le travail avec les personnes d'origine étrangère usant de violence au sein du couple renvoie apparemment les intervenants sociaux à un dilemme spécifique. S'ils entendent respecter les différences et les normes culturelles, ils entendent dans le même temps refuser celles qui légitimeraient des comportements violents. Cela conduit à des paradoxes et complexifie le travail, puisqu'il s'agit d'amorcer une approche multiculturelle tout en interdisant le recours à la violence. II en résulte, selon certains, le " risque de différences dans les messages, alors que les messages devraient être uniformes " face aux personnes usant de violence. Dans la pratique, cette situation se traduit par une difficulté à se positionner face à elles, avec le risque de ne pas clarifier « sa » zone de tolérance face à l'acte violent. La volonté du « respect des spécificités culturelles » tendrait alors à " relativiser certains actes de violence, une tendance qui caractérise la société ».

Les participants relient ces difficultés à un manque de formation dans ce champ : ils qualifient d'échec le fait que le travail avec les personnes usant de violence au sein du couple (et plus particulièrement celles d'origine étrangère) 
ne figure pas au nombre des offres de formation existantes. Dès lors, la collaboration avec les spécialistes de cette question devient un enjeu essentiel pour combler les lacunes en matière d'information.

\section{Des difficultés à prendre position}

Le manque de ressources et de soutien ainsi que le climat caractérisant certains débats politiques ont fait redouter à certains d'aborder le thème du recours à la violence conjugale par des membres de communautés étrangères. Ils craignent de renforcer à leur tour la stigmatisation de ces groupes. Ainsi, « certaines statistiques sont passées sous silence, par peur d'alimenter le discours politique » qui associe "recours à la violence » et «appartenance culturelle ». Mettre en parallèle violences et parcours migratoire s'érige en tabou. Ce qu'observent plusieurs intervenants sociaux, c'est que ces appréhensions se traduisent par un désengagement des professionnels du social dans le débat public. Ce retrait s'érige en obstacle majeur à l'élaboration de mesures de lutte contre les violences relationnelles adaptées aux groupes de migrants et à l'élaboration d'interventions orientées vers un changement de comportement.

\section{Une accessibilité réduite aux groupes thérapeutiques}

De manière générale, plusieurs professionnels relèvent un manque d'engagement systématique dans un groupe thérapeutique de la part des personnes usant de violence au sein du couple, en comparaison du nombre de victimes qui s'adressent à des centres d'hébergement. L'absence de mesures contraignantes, associée au fait que les groupes thérapeutiques exigent des participants un degré élevé d'introspection et des compétences cognitives, sociales et langagières minimales, rendraient ces groupes "peu accessibles ». Ils s'adresseraient en priorité aux personnes disposant de bonnes ressources intellectuelles, ce qui expliquerait la faible participation au programme vaudois. De plus, les séances se déroulant en français, la participation de personnes d'origine étrangère maîtrisant mal cette langue s'avère compromise. À cette difficulté s'ajoutent les appréhensions liées à l'autorisation de séjour - et au statut juridique associé - et à un statut socioéconomique souvent précaire. L'ensemble de ces facteurs dissuaderait les migrants de s'adresser à un tel service : comme l'évoque une intervenante, "les programmes ne sont pas accessibles aux migrants, et ce n'est pas qu'une question de la langue » mais parce qu'on observe " un [second] frein dans l'accès aux programmes pour les migrants: les craintes face aux conséquences juridiques, craintes [qui] dissuadent de faire des démarches $»$.

Sans le soutien d'interprètes ou l'existence de groupes thérapeutiques spécifiquement pour migrants, il est difficile, pour certains intervenants sociaux, d'inciter les personnes usant de violence au sein du couple à entreprendre une démarche auprès d'un service spécialisé. L'orientation vers le groupe thérapeutique devient alors l'exception plutôt que la règle. Les spécialistes dans l'intervention auprès des personnes usant de violence au sein du couple tendent, quant à eux, à solliciter les compétences de thérapeutes du réseau "sensibilisés à la question de la violence conjugale " lorsque l'intégration au groupe est impossible faute de maîtrise de la langue. Ces spécialistes proposent cette alternative en raison de " l'insuffisance des moyens pour assurer un suivi individualisé pour les migrants » et y voient une forme d'échec. En effet, ces personnes ne bénéficieront alors ni d'une intervention ciblée sur la violence, ni de la dynamique d'un groupe.

\section{L'intervention auprès des migrants usant de violence au sein du couple : un champ à renforcer}

Lors des groupes de discussion, les priorités dégagées ont notamment concerné la question de l'intervention spécifique auprès de personnes d'origine étrangère usant de violence au sein du couple. Les pistes évoquées ont visé en premier lieu l'intensification de la collaboration avec des acteurs clés comme les associations qui s'engagent dans la défense des droits des migrants, en s'inspirant des pratiques existantes pour les victimes. Travailler avec ces partenaires aurait pour objectif de développer des mesures sur trois niveaux: des campagnes de prévention ciblées, des projets de prévention en milieu scolaire et des stratégies pour faciliter l'intégration aux groupes thérapeutiques.

\section{Des messages de prévention destinés aux communautés étrangères}

Concrètement, il s'agirait de mener des campagnes dans les lieux fréquentés par des hommes appartenant à certaines communautés étrangères, tels que les "clubs sportifs ou de foot, entreprise, cantine d'entreprise » et 
dont les contenus respecteraient les différences culturelles. Les participants aux groupes de discussion ont proposé d'agir "en amont", considérant comme indispensable une collaboration étroite avec les milieux associatifs proches des populations migrantes. Il faudrait selon eux considérer les représentants des différentes communautés comme des partenaires à part entière pour améliorer l'accessibilité et la diffusion des messages préventifs, en mobilisant leurs savoir-faire et leurs réseaux lors de la conception des campagnes de prévention, du choix des espaces de diffusion et de la transmission des messages. Dans cette perspective, les craintes face au risque de stigmatisation des populations migrantes mériteraient une attention toute particulière.

Ont émergé aussi des propositions de développement, en complément des programmes existants, de projets en milieu scolaire ciblés sur les relations amoureuses violentes. En institutionnalisant un projet du type "Attitude respect ", il serait possible d'introduire par la suite la thématique des relations violentes. Ces projets informeraient sur la violence et expliqueraient aux jeunes la différence entre violence défensive et offensive. Les messages devraient aussi mettre en évidence que le recours à la violence n'est pas acceptable, y compris dans un cadre familial. Un volet particulier devrait être consacré aux garçons appartenant à certaines communautés étrangères, afin de susciter à cette occasion une réflexion au sujet des " messages culturels à caractère machiste ». L'objectif serait de "montrer que ces comportements inacceptables sont légitimés dans certaines familles et mettent des femmes dans des situations très difficiles $"$.

\section{Des collaborations pour améliorer l'accessibilité aux programmes thérapeutiques}

Face au constat que l'ensemble des personnes usant de violence au sein du couple n'accède pas aux groupes thérapeutiques, des membres du groupe de discussion ont recommandé de formaliser une collaboration avec l'association spécialisée dans l'intervention auprès des populations d'origine étrangère et avec des interprètes communautaires. Cette "collaboration avec Appartenances [doit favoriser l'] intervention auprès des auteurs " d'actes de violence conjugale ainsi que l'accessibilité aux groupes thérapeutiques pour ces derniers. En sus de la volonté de trouver des solutions aidant à dépasser les barrières linguistiques, ce travail interdisciplinaire permettrait de renforcer les compétences des intervenants sociaux et d'élaborer des pistes favorisant l'entrée en relation avec les personnes usant de violence au sein du couple issues des différentes communautés, tout en envisageant une intervention globale à partir d'une "approche multiculturelle autour de la définition de la personne auteure » d'actes de violence conjugale. Un autre objectif consiste à « mettre en relation les structures non spécialisées avec les associations » œuvrant dans le champ de la migration.

\section{Développer les compétences professionnelles dans deux directions}

À lire les résultats collectés lors de nos groupes de discussion, l'intervention auprès des personnes d'origine étrangère usant de violence au sein du couple reste difficile. Ont été relevées plusieurs limites au niveau de l'information disponible et des occasions de collaboration avec les milieux associatifs. A été abordée également la question de la posture à adopter face aux personnes appartenant à différentes communautés : comment associer le respect des spécificités culturelles tout en transmettant un message sans équivoque quant à l'interdit du recours à la violence? Au-delà de cette difficulté de positionnement dans leur pratique, les intervenants ont estimé que les possibilités d'intégrer un groupe thérapeutique étaient restreintes pour ces populations, et ce, pas uniquement en raison de l'exigence de la maîtrise du français. Cela limite pour elles les offres en matière d'orientation car elles ne bénéficient pas de ce type d'intervention, ce qui induit, du point de vue des intervenants, une forme d'inégalité.

Les professionnels du champ social peinent à se positionner, dans le débat public qui aborde la question migratoire, sous l'angle des besoins des personnes d'origine étrangère usant de violence au sein du couple ainsi que sous l'angle de la recherche de stratégies d'interventions ciblées. Ils craignent d'alimenter un discours stigmatisant, créant un lien de causalité directe entre origine socioculturelle et violence au sein du couple. Ils soulignent la nécessité de renforcer les collaborations avec les milieux associatifs en contact avec les populations migrantes, de recourir à des mesures adaptées, d'améliorer l'accès aux groupes thérapeutiques et finalement de s'engager activement dans un débat public.

Les dilemnes mis en évidence par l'analyse des données découlent d'une superposition de représentations, celles associées à la personne usant de violence au sein du couple (adoption de comportements socialement 
inacceptables en lien avec l'appartenance socioculturelle) et celles suscitées par le refus d'un discours politique discriminatoire à l'égard des minorités. Dès lors, l'intervenant se trouve face à un paradoxe susceptible de fragiliser à la fois son positionnement et la relation professionnelle qu'il cherche à créer : comment réussir à intervenir de manière spécifique auprès d'une personne d'origine étrangère qui a commis un acte répréhensible et accepter de la soutenir dans son processus d'intégration, sans ethniciser son comportement et en prévenant la stigmatisation?

Nos constats témoignent de l'importance à renforcer les pratiques à l'égard des migrants usant de violence au sein du couple, pratiques qui tiennent compte à la fois du contexte migratoire, de la vulnérabilité engendrée par la trajectoire de vie, de la violence elle-même et des obstacles à l'accès au réseau de soutien spécialisé. Cette double articulation - contexte migratoire et violence - nécessite de développer des compétences professionnelles spécifiques.

\section{Des pratiques qui tiennent compte du contexte migratoire...}

Concernant la dimension migratoire, la faible accessibilité au réseau spécialisé de soutien pour les migrants usant de violence au sein du couple relevée dans notre étude fait écho aux difficultés identifiées par diverses autres études traitant des réticences et des obstacles auxquels se heurtent les victimes migrantes. II s'agit notamment des craintes liées au statut administratif précaire et au risque de précarisation (Hargrove, 2006; Puig, 2002), de la méconnaissance du réseau (Egger et Schär Moser, 2008; Gillioz et collab., 1997; Vasconcelos 2005) et des barrières linguistiques (Burquier et collab., 2009; Crenshaw, 2005). Le décalage entre les pratiques professionnelles du pays d'accueil et celles découlant du modèle de référence et d'intervention de la culture d'origine suscite également une série de blocages (Cattaneo et Dal Verme 2009; Crenshaw, 2005).

Outre la connaissance des cadres légaux et administratifs, disposer de savoirs au sujet de la culture et du parcours migratoire s'avère essentiel pour cerner les appréhensions et les contraintes auxquelles font face les migrants, mais aussi les possibilités qui s'offrent à eux (Hargrove, 2006; Hénocque, 2004; Herla, 2008). Des connaissances de cet ordre permettent d'envisager des soutiens très concrets et de développer des pratiques respectueuses des normes et références culturelles (Herla, 2008). Elles offrent aussi des clés de lecture devant certaines réticences face aux pratiques thérapeutiques (Cattaneo et Dal Verme, 2009), ou encore devant des traumatismes résultant du parcours migratoire et de la violence subie (Batista Wiese et collab., 2009; Hargrove, 2006; Puig, 2002). Une intervention ciblée sur la violence exercée à l'égard du partenaire doit tenir compte de ces multiples dimensions, ceci afin de renforcer les capacités de résilience face aux difficultés rencontrées en situation de migration, y compris celles qui résultent de l'évolution de la cellule familiale et des rapports de pouvoir redéfinis entre membres du couple.

Au-delà des besoins en savoirs spécifiques, plusieurs recherches ont permis de souligner l'importance pour les intervenants de prendre conscience que leurs pratiques à l'égard des personnes d'origine étrangère ne sont pas exemptes de références culturelles et de préjugés. Il s'agit d'abord d'accepter cet état de fait (Herla, 2008). Ensuite, en se décentrant, l'intervenant peut tenir compte du cadre de référence de la personne d'origine étrangère et mobiliser les ressources de cette dernière (Hénocque, 2004). Le travail avec les membres d'une communauté étrangère exige donc de la créativité de la part de l'équipe, en acceptant notamment d'aller à la rencontre de ces personnes dans des contextes non usuels (Hargrove, 2006) ou encore de combiner différentes approches (Bolzman, 2009).

\section{... et du recours à la violence}

Selon nous, seule la combinaison de mesures judiciaires (rappel de l'interdit du recours à la violence) et de mesures thérapeutiques conduit à la cessation durable de la violence au sein du couple. Toutefois, en Suisse, la proportion de personnes usant de violence au sein du couple qui entreprennent une démarche auprès d'un service spécialisé reste faible (Egger, 2008). Les mécanismes de neutralisation (Levesque, Velicer, Castel et Greene, 2008; Mayer, 2007) qui permettent aux auteurs d'actes de violence conjugale à la fois de minimiser leur responsabilité ainsi que les risques de récidive encourus par la victime et de restaurer leur cohérence interne (ne pas se voir comme agresseur, mais comme victime) freinent leur implication dans le travail thérapeutique. Lorsqu'ils sollicitent un soutien auprès d'un service spécialisé, leur demande a une valeur principalement instrumentale: rassurer la victime, maintenir la relation ou répondre à des exigences externes (Lorenz et Anglada, 2011). Face à ces 
réticences, nous pensons que les intervenants sociaux peuvent non seulement identifier les situations de violence conjugale, mais aussi susciter une réflexion pour accompagner les personnes qui en sont à l'origine à prendre contact en vue d'une intervention spécialisée. Toutefois, les professionnels du champ social semblent restreindre leurs pratiques, en recourant à ce que Hofner et Mihoubi-Culand (2008) appellent le " tout faire " ou « ne rien proposer » : soit ils renoncent à orienter et s'en remettent à la magistrature pour " contraindre » l'auteur d'actes de violence conjugale, soient ils ne passent pas le flambeau aux spécialistes et assurent eux-mêmes un suivi.

\section{En conclusion : des propositions d'amélioration selon trois axes}

Ces constats nous amènent à proposer des stratégies face aux personnes migrantes usant de violence au sein du couple qui relèvent de plusieurs axes.

Développer des procédures de détection et d'orientation des personnes usant de violence au sein du couple en intégrant une approche ethnoculturelle

Les protocoles de détection ont fait leur preuve dans le travail avec les victimes. Ils font toutefois cruellement défaut lorsqu'il est question de personnes usant de violence au sein du couple, de surcroît étrangères. Ces outils donnent aux professionnels les moyens de repérer cette violence, leur offrent des repères concrets pour identifier les personnes concernées, les aident à surmonter les appréhensions et facilitent leur positionnement professionnel lors de l'orientation (Hegarty, Taft et Feder, 2008; Hofner et Mihoubi-Culand, 2008). Les pratiques d'orientation, tout en transmettant l'information, visent à réduire le décalage entre la responsabilité que les personnes usant de violence au sein du couple sont prêtes à assumer et les exigences associées au travail avec un groupe thérapeutique. L'intervention consiste alors à convaincre les individus qu'ils ont à y gagner en s'engageant dans une démarche thérapeutique et à diminuer leurs craintes face à d'éventuelles conséquences négatives (expulsion, stigmatisation). Il s'agit, au cours de cette phase, de reconnaître l'appartenance culturelle des personnes usant de violence au sein du couple et de "s'appuyer sur les valeurs de la culture d'origine des populations concernées" (Bolzman, 2009, p.45) pour qu'elles sollicitent ou acceptent de l'aide visant à prévenir le recours aux comportements violents.

\section{Renforcer les pratiques communautaires au moyen de mesures d'intervention visant les communautés étrangères en étroite collaboration avec les milieux concernés}

Ce renforcement (Bolzman, 2009) vise à surmonter les difficultés liées au parcours migratoire (fin de l'isolement, création de liens et de capital social, partage des ressources, etc.) en partant de la culture d'origine, mais aussi à informer au sujet des normes en vigueur (interdit du passage à l'acte, droit au soutien, etc.). Cet axe doit favoriser les pratiques permettant l'accès et l'implication des personnes migrantes usant de violence conjugale aux mesures thérapeutiques spécialisées, notamment grâce à la présence d'interprètes communautaires formés dans le champ de la violence au sein du couple. Néanmoins, il faut éviter de créer des mesures thérapeutiques réservées aux personnes d'origine étrangère, afin de prévenir toute stigmatisation et toute limitation des échanges interculturels. À titre d'exemple, la richesse des groupes thérapeutiques pour personnes usant de violence conjugale réside justement dans le partage avec des membres d'origines socioculturelles et de parcours différents (Lorenz et Anglada, 2011). S'engager dans un débat public afin de promouvoir les conditions encadrant la création de pratiques
d'intervention adaptées aux personnes migrantes dans une perspective antidiscriminatoire

Cet engagement consiste pour les professionnels à accepter, certes, un lien potentiel entre des formes de violence conjugale et un contexte migratoire, mais en même temps à réfléchir en termes de facteurs de risque, de reconnaissance de besoins spécifiques liés à une certaine vulnérabilité et de volonté de combattre une forme d'inégalité dans l'accès aux mesures thérapeutiques (Bolzman, 2009). Il s'agit alors, pour les intervenants du champ social, de s'engager pour l'élaboration d'une politique publique qui ne se limite pas aux seules réponses répressives et à l'expulsion des migrants auteurs d'actes de violence conjugale.

Alterstice - Revue Internationale de la Recherche Interculturelle, vol. 3, $n^{\circ} 2$ 
En conclusion, nous sommes d'avis que l'intervention sociale auprès des migrants usant de violence au sein du couple nécessite le développement de pratiques qui se situent à l'intersection de trois modèles: l'approche ethnoculturelle, l'approche communautaire et l'approche antidiscriminatoire. En associant ces approches, il devient possible de limiter les effets pervers cités par Bolzman (2009) : le relativisme culturel (ne voir dans l'acte violent qu'un problème d'appartenance socioculturelle), la ghettoïsation voire la stigmatisation des migrants usant de violence au sein du couple (ne pas favoriser par exemple les échanges interculturels) ou, enfin, la minimisation des facteurs culturels et personnels (ne s'intéresser qu'aux conditions de vie liées à la migration).

\section{Références bibliographiques}

Acheroy, C. et Hadjaj-Castro, H. (2006). La méthode SEPO/SWOP. Bruxelles : COTA ASBL.

Anglada, C. et Lorenz, S. (2007). Le traitement des auteurs de violence : contraintes privée et publique. Dans Institut pour l'égalité des femmes et des hommes, Les hommes et l'égalité (p. 97-102). Bruxelles : Institut pour l'égalité des femmes et des hommes.

Barz, M. et Helfferich, C. (2006). Häusliche Gewalt beenden: Verhaltensänderung von Tätern als Ansatzpunkt. Vorgehen und Wirkung von Täterprogrammen im Kontext von Interventionsprojekten gegen häusliche Gewalt in Baden-Württemberg. Stuttgart : Landesstiftung Baden-Württemberg

Batista Wiese, E., Van Dijk, M. et Seddik, H. (2009). La matrice familiale dans l'immigration : trauma et résilience. Dialogue, 185(3), 67-78.

Belser, K. (2005). La violence domestique survient dans tous les milieux - mais dans certains peut-être un peu plus souvent. Questions au féminin, 1, 13-16. (Violence domestique et migration)

Black, D., Schumacher, A., Smith, A. et Heyman, R. (1999). Partner, child abuse risk factors literature review [en ligne]. National Network on Family Resiliency, National Network for Health. www.nnh.org

Bolzman, C. (2009). Modèles de travail social en lien avec les populations migrantes : enjeux et défis pour les pratiques professionnelles. Pensée plurielle, 2(21), 41-51.

Broué, J. et Guévremont, C. (1999). Intervenir auprès des conjoints violents. Québec : Éditions Saint-Martin.

Bureau fédéral de l'égalité entre femmes et hommes (BFEG) (2012). Feuille d'information : La violence domestique dans le contexte de la migration [en ligne]. Berne : Bureau fédéral de l'égalité entre femmes et hommes. http://www.ebg.admin.ch/dokumentation/00012/00442/index.html?lang=fr

Burquier R., Hofner M.-C., Romain N. et Mangin P. (2009). Caractéristiques des femmes victimes de violence graves dans un échantillon clinique. Journal International de victimologie 21, 7(3), 4-11.

Collectif ViFa. (2000). Violence et famille intervient auprès d'hommes ayant recours à la violence conjugale et domestique. (Document non publié)

Cattaneo, M. et Dal Verme, S. (2009). Conflits familiaux autour de la maternité dans la migration. Dialogue, 185(3), 79-89.

Crenshaw, K. (2005). Cartographies des marges: intersectionnalité, politique de l'identité et violences contre les femmes de couleur. Cahiers du genre, 39, 51-82.

Damba, R. et Anglada, C. (2005). Violence conjugale et hommes migrants : quelles perspectives? Violences domestiques et migration, questions au féminin, 1, 55-57.

Egger, T. (2008). Travail de consultation et programmes de lutte contre la violence destinés aux auteur-e-s de violences conjugales en Suisse. Berne : Bureau fédéral de l'égalité entre femmes et hommes, Service de lutte contre la violence.

Egger, T. et Schär Moser, M. (2008). La violence dans les relations de couple. Ses causes et les mesures prises en Suisse. Berne: Bureau fédéral de l'égalité entre femmes et hommes, Service de lutte contre la violence.

Flick, U. (2000). Qualitative Forschung. Ein Handbuch. Hamburg : Rowohlt Verlag. 
Garcia Moreno, C. (2003). La violence de genre : une vision d'ensemble. Dans L. Gillioz, R. Gramoni, C. Margairau et C. Fry (dir.), Voir et agir. Responsabilité des professionnel-le-s de la santé en matière de violence à l'égard des femmes. Genève : Médecine et hygiène. (Cahiers médico-sociaux)

Gillioz, L., De Puy, J.et Ducret, V. (1997). Domination et violence envers la femme dans le couple. Lausanne : Payot.

Gloor, D. et Meier, H. (2004). Frauen, Gesundheit und Gewalt im sozialen Nahraum. Repräsentativbefragung bei Patientinnen der Maternité Inselhof Triemli, Klinik für Geburtshilfe und Gynäkologie, Zürich, im Auftrag des Gleichstellungsbüros der Stadt Zürich und der Maternité Inselhof Triemli. Berne : Edition Soziothek.

Godenzi, A. et Yodannis, C. (1999). Report on the economic costs of violence against women. Fribourg : Université de Fribourg.

Gondolf, E. (2003). Evaluating batterer counseling programs: a difficult task showing some effects and implications. Aggression and Violent behavior, 9, 605-631.

Hargrove, P. (2006). Social Work Practice with Mexican Clients. Journal of Emotional Abuse, 6(2-3), 61-76.

Hegarty, K., Taft, A. et Feder, G. (2008). Violence between intimate partners: working with the whole family. $B M J$, $337,337-351$.

Hénocque, C. (2004). Travail social et migration familiale. Informations sociales, 113, 92-99.

Herla, R. (2008). Travail social, violence conjugale et multiculturalité. Liège : Collectif contre les violences familiales et exclusion.

Hirigoyen, M.-F. (2005). Femmes sous emprise. Les ressorts de la violence dans le couple. Paris : Oh Édition.

Hirigoyen, M.-F. (2009). De la peur à la soumission. Empan, 73(1), 24-30. (nº spécial : Les violences conjugales)

Hofner, M.-C. et Mihoubi-Culand, S. (2008). Le rôle des professionnel(le)s de l'action médico-sociale dans la prévention de la violence conjugale. Questions au féminin, 2, 90-95.

Hofner, M.-C., Stalder, C., Pedevilla, L., Detraz, J. et Saturno, A. (2011). 10 ans de lutte contre la violence domestique dans le canton de Vaud. Résumé de la recherche menée par l'unité de Médecine des violences sur le mandat de la Commission cantonale de lutte contre la violence domestique. Lausanne : Bureau de l'égalité entre les femmes et les hommes Vaud.

Jaquier, V. (2008). Prise en charge policière et judiciaire des violences domestiques : Méthodologie d'une première recherche exploratoire et principaux résultats. Revue internationale de criminologie et de police technique et scientifique, 61(4), 403-428.

Jaspard, M. (2005). Les violences contre les femmes. Paris : La Découverte.

Johnson, M. (2005). Domestic violence: it's not about gender - or is it? Journal of Marriage and Family, 67(5), 11261130.

Killias, M. (2002). Précis de criminologie. Berne : Stampfli.

Killias, M., Simonin, M. et De Puy, J. (2005). Violence experienced by women in Switzerland over their lifespan: Results of the International Violence against Women Survey (IVAWS). Berne : Stampfli.

Krug, E. G., Dahlberg, L. L., Mercy, J. A., Zwi, A. et Lozano-Ascencio, R. (dir.) (2002). Rapport mondial sur la violence et la santé. Genève : Organisation mondiale de la santé.

Levesque, D., Velicer, W., Castel, P. et Greene, E. (2008) Resistance Among Domestic Violence Offenders. Measure Development and Initial Validation. Violence against women, 14(2), 158-184.

Lorenz S. et Bigler P. (2013). Responsabilisation et dévoilement : le rôle d'un programme pour hommes auteurs de violences au sein du couple, Pensée plurielle, 1(32), 115 -127.

Lorenz, S. et Anglada, C. (2011). Favoriser le changement chez des auteurs de violence dans le couple: le rôle du travail de groupe. Revue de FESET - Journal européen de l'Éducation sociale, 73-89.

Alterstice - Revue Internationale de la Recherche Interculturelle, vol. 3, $n^{\circ} 2$ 
Mathieu, C., Bélanger, C. et Bribois, H. (2006). Thérapie de groupe pour hommes violents envers leur conjointe : abandon thérapeutique chez ces hommes. Santé mentale au Québec, 31, 169-187.

Mayer, K. (2007). Männer, die Gewalt gegen die Partnerin ausüben. Dans Fachstelle für Gleichstellung Stadt Zürich, Frauenklinik Maternité, Stadtspital Triemli, Zürich, Verein Inselhof Triemli (dir.), Häusliche Gewalt erkennen und richtig reagieren. Handbuch für Medizin, Pflege und Beratung (p. 65-75). Berne : Verlag Hans Huber.

Mösch Payot, P. (2008). La situation juridique actuelle en matière de violence domestique en Suisse : innovations, contexte, questions. Questions au féminin, 2, 22-27.

Myers, K. (1996). Sommaire des projets de recherche et développement entrepris par les affaires correctionnelles en matière de violence conjugale. Ottawa : Solliciteur général du Canada.

OFAS - Office Fédéral des Assurances Sociales (2012). Monitorage AS-Al-AC : Les indicateurs de base $2005-2010$ [en ligne]. http://www.bsv.admin.ch/dokumentation/zahlen/00095/03084/index.html?lang=fr

Paradis, L. (2012). L'enfant, une éponge... L'enfant exposé à la violence conjugale. Son vécu, notre rôle. Québec: Direction régionale de santé publique de la Capitale-Nationale.

Puig, M. E. (2002). The Adultification of Refugee Children. Journal of human behavior in the social environment, 5(34), 85-95.

Rojas-Viger, C., (2008). L'impact des violences structurelle et conjugale en contexte migratoire : perceptions d'intervenants pour le contrer. Nouvelles pratiques sociales, 20(2), 124-141.

Romito, P. (2003). Comment les services sociaux et de santé répondent-ils aux besoins des femmes violentées. Dans L. Gillioz, R. Gramoni, C. Margairau et C. Fry (dir.), Voir et agir. Responsabilité des professionnel-le-s de la santé en matière de violence à l'égard des femmes. Genève : Médecine et hygiène. (Cahiers médicosociaux)

Rondeau, G., Brodeur, N. et Carrier, N. (2001). L'intervention systémique et familiale en violence conjugale fondements. Modalités, efficacité et controverse. Montréal et Québec : Centre de recherche interdisciplinaire sur la violence familiale et la violence faite aux femmes (CRI-VIFF). (Études et analyse $n^{\circ} 16$ )

Rossel, R., Sorenti, I. et Jaquier, V. (2007, juillet) La violence domestique portée à la connaissance de la Police cantonale vaudoise, Crimiscope, 35.

Saunders, D. G. et Parker, J. C. (1989). Legal sanction and treatment follow-through among men who batten: A multivariate analysis. Social Work Research and Abstracts, 25(3), 21-29.

Seith, C. (2003) Les réponses des institutions dans les situations de violence conjugale. L'exemple de la police, des services sociaux et de la santé. Dans L. Gillioz, R. Gramoni, C. Margairau et C. Fry (dir.), Voir et agir. Responsabilité des professionnel-le-s de la santé en matière de violence à l'égard des femmes. Genève: Médecine et Hygiène. (Cahiers médico-sociaux)

Schwander, M. (2006). Violence domestique : analyse juridique des mesures cantonales. Berne : Bureau fédéral de l'égalité entre femmes et hommes.

Séverac, N. (2009). Auteurs de violence conjugale: Sanction / éducation, deux points d'appui pour sortir de la violence. Revue Empan, Les violences conjugales, 73, 103-109.

Silvestre, M., Heim, C. et Christen, M. (1999). Du traitement de la violence conjugale. Thérapie familiale, 20(4), 403424.

Steiner, S. (2004). Ausländerkriminalität am Beispiel der Häuslichen Gewalt. Kriminalistik, 58, 717-720.

Vasconcelos, M. L. (2005). Campagnes de prévention des violences faites aux femmes auprès des populations migrantes à Genève. Questions au féminin, 1, 51-52.

Walker, L. (2000). The Battered Women Syndrome. New York : Springer Publishers. 
Zoder, I. (2008). Homicide dans le couple. Affaires enregistrées par la police de 2000 à 2004. Neuchâtel : Office fédéral de la statistique. 